\title{
SCATTERING OF EXCITONS BY PHONONS IN QUANTUM WELLS*
}

\author{
W. Bardyszewsiki and M. Prywata \\ Institute of Theoretical Physics, Warsaw University, Hoża 69, 00-681 Warsaw, Poland
}

A method to describe the effects of the exciton-optical phonon interaction is presented using the cumulant expansion approximation. The functional-integral technique of colherent phonon states is used in order to justify the commonly used model Hamiltonian and generate the proper perturbation series. The influence of the mutual electron-hole screening on the polaronic effects in quantum wells is analyzed.

PACS numbers: 73.20.Dx, 71.38.+i, 72.80.Ey

\section{Introduction}

The most prominent features in the band-to-band optical absorption spectra in semiconductor quantum-well systems are related to excitons. Consequently, it is very important to understand the influence of the exciton-phonon coupling on the excitonic line shape. In ionic crystals usually the polar interaction of charged particles with longitudinal optical phonons plays the dominant role. Such coupling however, should be very small for small excitons in which both charges are confined to a very limited region in space, effectively screening each other. In the opposite limit of large exciton radius, the coupling of both particles with phonons is more or less independent leading to stronger renormalizations. The transition between these two regimes is set roughly by the relationship between the exciton and individual polaron radii. In this article we extend the cumulant expansion method which was previously employed for the single particle case [1] to excitons in quantum wells.

\section{Exciton polaron Hamiltonian}

According to the linear response theory the absorption spectra in the vicinity of the energy gap in semiconductors is determined by the electron-hole pair correlation function, which for the imaginary time is represented by the time ordered product

$$
P\left(1^{\prime} 2^{\prime}, 12\right)=-\left\langle T\left[\mathcal{A}\left(1^{\prime} 2^{\prime}\right) \mathcal{A}^{\dagger}(12)\right]\right\rangle
$$

*This work has been partially supported by the grant 2 P302 16506 of the Committee for Scientific Research. 
of the pair annihilation and creation operators $\mathcal{A}\left(1^{\prime} 2^{\prime}\right) \equiv \psi^{\dagger}\left(1^{\prime}\right) \psi\left(2^{\prime}\right)$ and $\mathcal{A}^{\dagger}(12) \equiv$ $\psi^{\dagger}(2) \psi(1)$ at times $\tau^{\prime}$ and $\tau$ respectively, expressed in terms of the electron creation and annihilation operators $\psi^{\dagger}$ and $\psi$.

The expression for $P\left(1^{\prime} 2^{\prime}, 12\right)$ may be written in the path integral form with respect to the phonon coherent states $b_{q}^{*}$ and $b_{q}$ :

$$
P\left(1^{\prime} 2^{\prime}, 12\right)=\frac{1}{Z} \int \mathcal{D}\left[b_{q}^{*} b_{q}\right] \mathrm{e}^{-S_{\mathrm{ph}}} \mathcal{G}\left(1^{\prime} 2^{\prime}, 12, b_{q}^{*} b_{q}\right)
$$

where the effective action for phonons is taken in the harmonic approximation as

$$
S_{\mathrm{ph}}(\tau)=\sum_{\boldsymbol{q}}\left[b_{\boldsymbol{q}}^{*} \frac{\partial}{\partial \tau} b_{\boldsymbol{q}}+\hbar \omega_{\boldsymbol{q}}\left(b_{\boldsymbol{q}}^{*} \boldsymbol{b}_{\boldsymbol{q}}+\frac{1}{2}\right)\right]
$$

and $\mathcal{G}\left(1^{\prime} 2^{\prime}, 12, b_{q^{*}}^{*} q\right)$ denotes the electron-hole pair correlation function for the particular path $b q(\tau)$. Thus Eq. (2) describes an averaged pair correlation function over all possible evolutions of the phonon system, precisely as in the polaron theory.

The linearized equation for $\mathcal{G}$ in the mean field approximation corresponds to the evolution of an exciton in the time dependent potential due to phonon fluctuations. The corresponding Hamiltonian consists therefore of a part for the unperturbed exciton at lattice equilibrium and of the exciton-phonon coupling term which is treated as a perturbation. Using the path integral representation of Eq. (2) one can easily generate the perturbation series expansion for $P$. It turns out that the identical series is generated assuming that the pair operators $\mathcal{A}$ and $\mathcal{A}^{\dagger}$ represent bosons in the Hamiltonian

$$
\begin{aligned}
H= & \sum_{p_{\alpha}} E_{p_{\alpha}} \mathcal{A}_{p_{\alpha}}^{\dagger} \mathcal{A}_{p_{\alpha}}+\sum_{q} \hbar \omega_{q}\left(b_{q}^{\dagger} b_{q}+\frac{1}{2}\right) \\
& +\sum_{\boldsymbol{q} p} \sum_{\alpha \beta} \mathcal{A}_{\boldsymbol{p}+\boldsymbol{q}_{\|}, \beta}^{\dagger} \mathcal{A}_{p_{\alpha}} V_{\boldsymbol{q}}^{\beta \alpha}\left(b_{q}+b_{-q}^{\dagger}\right)
\end{aligned}
$$

which can be treated as an effective exciton-phonon Hamiltonian only under the assumption of zero occupancy for excitons [2]. We have here specified to the quantum-well situation in which an exciton state with energy $E_{p_{\alpha}}$ is fully characterized by the total momentum $p$ parallel to the quantum-well plane and the internal quantum state label $\alpha$. The last term in Eq. (4) describes the interaction of excitons with bulk phonons and it is obtained from the three-dimensional Fröhlich Hamiltonian by taking expectation values with respect to the envelope functions in the direction perpendicular to the quantum-well plane [1].

\section{Spectral density function}

The probability of generating an exciton in the state $\alpha$ and with the total momentum $\boldsymbol{p}=0$ by absorbing a photon of frequency $\omega$ is proportional to the spectral density function

$$
A_{\alpha}(\omega) \equiv-\frac{1}{\pi} \operatorname{Im} P_{\alpha}^{\mathrm{ret}}(\omega)
$$

where the retarded Green function $P_{\alpha}^{\text {ret }}(\omega)$ is obtained from the Fourier transform of its temperature counterpart defined in Eq. (1) by the standard procedure of analytic continuation in the complex frequency plane [3]. It can be also obtained by 
solving the appropriate Dyson equation with the proper, retarded self-energy function. Unfortunately, the perturbation series expansion for the self-energy function is very difficult to obtain and, in most cases, converges very slowly. The situation can be improved by applying the cumulant expansion verified previously in single polaron problem in three dimensions [4]. Using the lowest order expression for the retarded self-energy function $\Sigma_{\alpha}^{(1)}(\omega)$ and neglecting its off-diagonal matrix elements we arrive at the following expression:

$$
P_{\alpha}^{\text {ret }}(t)=\exp \left[-\frac{1}{\pi} \int_{-\infty}^{\infty} \frac{\mathrm{d} \omega}{2 \pi} \frac{1-\mathrm{i} \omega t-\mathrm{e}^{-\mathrm{i} \omega t}}{\omega^{2}} \operatorname{Im} \Sigma_{\alpha}^{(1)}(\omega)\right] .
$$

The resulting Green function contains contributions to infinite order in the coupling constant. Such a procedure has proper limiting behavior for infinitely large mass of excitons and yields a reasonable description of multi-phonon processes for the finite mass.

\section{Results and discussion}

From the shape and position of the excitonic absorption line determined from the spectral density defined in Eqs. (5) and (6) one can infer the renormalization of the exciton energy and the lifetime for a particular state $\alpha$. Due to the composite nature of excitons we expect that these characteristics should depend on exciton structure. The fundamental parameter in this case is the exciton radius. We have accordingly evaluated the exciton ground state energy renormalization as a function of exciton radius. The results plotted in Fig. 1 were obtained by vary-

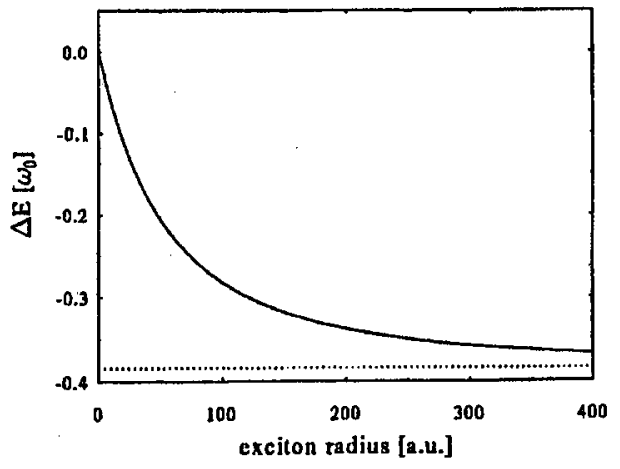

Fig. 1. The exciton ground state energy shift in the infinitely narrow GaAs/GaAlAs quantum well (solid line) compared to the case of decoupled electron-hole pair case (dotted line).

ing the radius of the hydrogen-like excitonic wave function assuming all the other parameters for GaAs/GaAlAs lattice matched quantum-well system, in the limit of infinitely narrow well. The value of the energy shift quite rapidly reaches the saturation value marked by the horizontal dotted line, corresponding to the limit of decoupled electron and hole. In fact, this transition to the independent polaron situation should occur much faster in the quantum wells due to the confinement of 


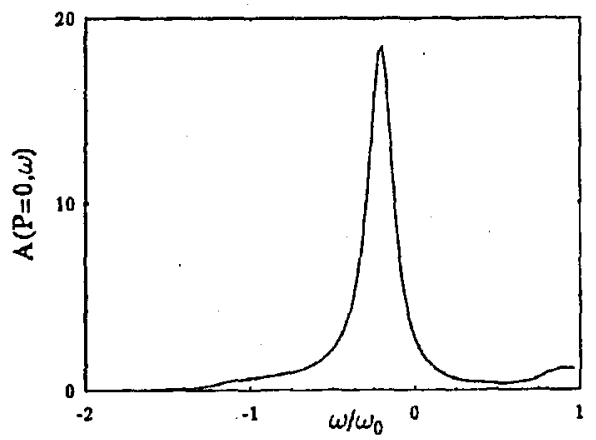

Fig. 2. Spectral density function of the exciton ground state in the GaAs/GaAlAs quantum well of width $L=100 \AA$ at temperature $T=300 \mathrm{~K}$.

charges, which generally amplifies the polaron effect. Thus the mutual screening of the electron and hole charges is much weaker in the quantum well than in the absence of confinement. The results presented in Fig. 1 are particularly sensitive to the method of approximation for the sum over finite states for exciton ionization processes. We have employed the technique suggested in Ref. [5] corrected by multiplying the final result by the factor of $\left(\sqrt{m_{\mathrm{h}}}+\sqrt{m_{\mathrm{e}}}\right) / \sqrt{m_{\mathrm{h}}+m_{\mathrm{e}}}$ in order to compensate for the systematic error introduced by that approximation. Similar factor is then applied to the imaginary part of $\Sigma$ in the cumulant expansion (5). The resulting curve for the spectral density function using the same set of parameters is presented in Fig. 2. According to our model the main excitonic line is asymmetric and has phonon satellites separated by the LO phonon energy. Even though the exciton has finite mass and recoil effects should lead to smearing of such structures, one can easily distinguish at least one satellite on each side of the line at $T=300 \mathrm{~K}$.

\section{Conclusions}

We have formulated exciton-polaron problem using the path integral formalism which allowed to generate a well-defined perturbation expansion for the exciton-phonon interaction. By applying the cumulant expansion we were able to model the excitonic line shape function. We have studied the transition from tightly bound to decoupled electron-hole pair regime in the typical quantum-well system. The spectral density function for the main absorption line for excitons is asymmetric and has phonon satellites similarly as in the case of localized exciton.

\section{References}

[1] W. Bardyszewski, M. Prywata, Acta Phys. Pol. A 88, 969 (1995).

[2] S. Rudin, T.L. Reinecke, Superlattices Microstruct. 3, 137 (1987).

[3] A.L. Fetter, J.D. Walecka, Quantum Theory of Many-Particle Systems, McGraw-Hill, San Francisco 1971.

[4] D. Dunn, Can. J. Phys. 53, 321 (1975).

[5] J. Liebler, H. Haug, Phys. Rev. B 41, 5843 (1990-II). 